\title{
Market-Based Multirobot Coordination: A Survey and Analysis
}

\author{
When robots work together as a team, the members that perform each task should \\ be the ones that promise to use the least resources to do the job.
}

By M. Bernardine Dias, Robert Zlot, Nidhi Kalra, and Anthony Stentz

\begin{abstract}
Market-based multirobot coordination approaches have received significant attention and are growing in popularity within the robotics research community. They have been successfully implemented in a variety of domains ranging from mapping and exploration to robot soccer. The research literature on market-based approaches to coordination has now reached a critical mass that warrants a survey and analysis. This paper addresses this need for a survey of the relevant literature by providing an introduction to marketbased multirobot coordination, a review and analysis of the state of the art in the field, and a discussion of remaining research challenges.
\end{abstract}

KEYWORDS | Auctions; market-based coordination; multirobot teams; resource allocation; task allocation

\section{INTRODUCTION}

As robots become an integral part of human life, we charge them with increasingly varied and difficult tasks including planetary exploration, manufacturing and construction, medical assistance, search and rescue, and port and warehouse automation. Like humans, robots working in challenging domains can potentially perform better by working together in teams than by working alone. Ideally, robots will coordinate to redistribute resources amongst themselves in a way that enables them to accomplish their mission efficiently and reliably. Coordination can lead to faster task completion, increased robustness, higher

Manuscript received June 1, 2005; revised June 1, 2006. This work was supported in part by the Boeing Company under Grant CMU-BA-GTA-1, in part by the U.S. Army Research Laboratory, Robotics Collaborative Technology Alliance, under Contract DAAD19-01-2-0012), and in part by the Qatar Foundation for Education, Science and Community Development.

The authors are with The Robotics Institute, Carnegie Mellon University, Pittsburgh, PA 15213 USA, and with Carnegie Mellon University in Qatar, Doha, Qatar (e-mail: mbdias@ri.cmu.edu; robz@ri.cmu.edu; nidhi@ri.cmu.edu; axs@ri.cmu.edu).

Digital Object Identifier: 10.1109/JPROC.2006.876939 quality solutions, and the completion of tasks impossible for single robots. However, these domains simultaneously present many obstacles to effective coordination, such as dynamic events, changing task demands, resource failures, the presence of adversaries, and limited time, energy, computation, communication, sensing, and mobility. Therefore, coordinating a multirobot team requires overcoming many formidable research challenges.

Humans have met these coordination challenges for thousands of years with increasingly sophisticated market economies. In these economies, self-interested individuals and groups trade goods and services to maximize their own profit; simultaneously, this redistribution results in an efficient production of output for the system as a whole. Researchers have recently applied the principles of market economies to multirobot coordination. In market-based multirobot systems, robots are designed as self-interested agents that operate in a virtual economy. Both the tasks that must be completed and the available resources are commodities of measurable worth that can be traded. For example, tasks can be assigned to robots via market mechanisms such as auctions. When a robot completes a task, it receives some payment in the form of virtual money for providing a service to the team. However, the robot must also pay for the resources it consumed to complete the task. The essence of market-based approaches is that, in a well-designed system, the process of robots trading tasks and resources with one another to maximize individual profit simultaneously improves the efficiency of the team.

To illustrate this more concretely, consider a team of robots performing a distributed sensing mission on Mars. As illustrated in Fig. 1, the robots must gather data from specific sites of interest to scientists while consuming the least amount of energy. One important aspect of completing the mission is to determine which robot should visit each site. We can solve this problem using a market-based 


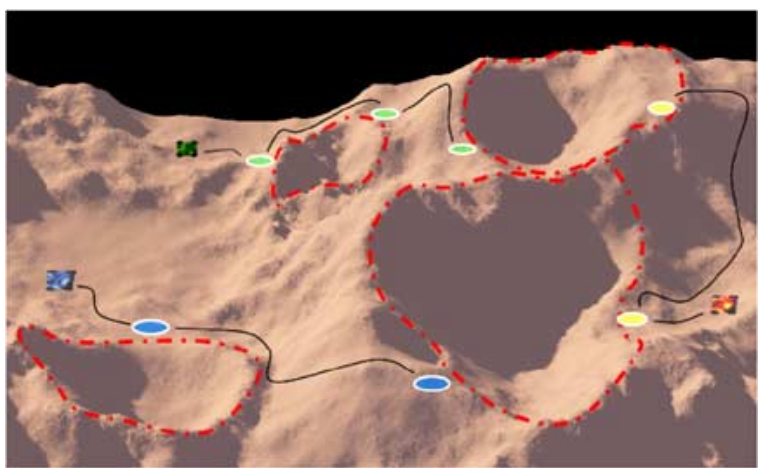

Fig. 1. An illustration of three robots exploring Mars. The robots' task is to gather data around the four craters, which can be achieved by visiting the highlighted target sites.

approach in which robots compete in auctions for each task of visiting a site. After estimating their resource usage for an offered task and submitting bids based on those expected costs, the robot with the best bid is awarded a contract for that site.

Suppose that we offer a maximum reward of $\$ 50$ for each task and that robots incur a cost of $\$ 2$ for each meter of travel (since the resource of concern is energy consumed). This $\$ 50$ is a reserve price that essentially says that the task should only be attempted if the site can be reached by increasing one's path length by less than $25 \mathrm{~m}$. Further suppose that a robot $A$ is only $5 \mathrm{~m}$ from a site $S$. Since $A$ would have to spend $\$ 10$ to complete the task, it bids $\$ 10$. Meanwhile, a robot $B$ that is $10 \mathrm{~m}$ from the site bids $\$ 20$. A is awarded the contract because it can perform the task more efficiently and for less than the reserve price.

This simple example illustrates the basic mechanism of a market-based approach to coordination. As the problem increases in complexity with the addition of more robots, more resources (e.g., time, network bandwidth, computing power, sensors, etc.), added constraints between the tasks, dynamically changing tasks, and so forth, the coordination approach requires added functionality to produce efficient solutions. We use this distributed sensing scenario throughout the remainder of the paper to illustrate the complexities of coordination and the diversity of marketbased approaches.

The earliest examples of market-based multiagent coordination appeared in the literature over 30 years ago [1], [2] and have been modified and adopted for multirobot coordination in more recent years. This paper is motivated by the growing popularity of market-based approaches and the lack of a comprehensive review of these approaches. This paper makes three contributions to the robotics literature. First, it provides a tutorial on market-based approaches by discussing the motivating philosophy, defining the requirements and tradeoffs inherent in such approaches, analyzing their strengths and weaknesses, and placing them appropriately in the context of the larger set of approaches to multirobot coordination. Second, this paper surveys and analyzes the relevant literature. Finally, it inspires and directs future research on this topic through a discussion of remaining challenges.

The scope of this paper is limited to market-based approaches for coordinating teams that include robots. Moreover, this review principally considers approaches that actively reason about the existence of other agents when coordinating the team, in contrast to approaches in which agents coexist. Nevertheless, related publications outside the stated scope of this paper are included as necessary to augment the discussion.

The following section provides an introduction to market-based mechanisms for readers less familiar with the field. This overview is followed by a extensive review of market-based multirobot coordination approaches to date, categorized and analyzed across several relevant dimensions: planning, solution quality, scalability, dynamic events and environments, and heterogeneity. The paper concludes with a summary of the survey and future challenges in this research area.

\section{OVERVIEW}

In this section, we discuss key concepts that will provide a foundation for the remainder of the paper, including a definition of market-based approaches and an introduction to auctions. We then place market-based approaches in the larger spectrum of coordination approaches.

\section{A. Definition of a Market-Based Approach}

Most market-based multirobot and multiagent coordination approaches share a set of underlying elements. Market theory provides precise definitions for several of these elements. Borrowing from both bodies of literature, we define a market-based multirobot coordination approach based on the following requirements.

- The team is given an objective that can be decomposed into subcomponents achievable by individuals or subteams. The team has access to a limited set of resources with which to meet this objective.

- A global objective function quantifies the system designer's preferences over all possible solutions.

- An individual utility function (or cost function) specified for each robot quantifies that robot's preferences for its individual resource usage and contributions towards the team objective given its current state. Evaluating this function cannot require global or perfect information about the state of the team or team objective. Subteam preferences can also be quantified through a combination of individual utilities (or costs).

- A mapping is defined between the team objective function and individual and subteam utilities (or costs). This mapping addresses how the individual 
production and consumption of resources and individuals' advancement of the team objective affect the overall solution.

- Resources and individual or subteam objectives can be redistributed using a mechanism such as an auction. This mechanism accepts as input teammates' bids, which are computed as a function of their utilities (or costs), and determines an outcome that maximizes the mechanism-controlling agent's utility (or minimizes the cost). In a welldesigned mechanism, maximizing the mechanismcontrolling agent's utility (or minimizing cost) results in improving the team objective function value.

\section{B. Auctions}

Auctions are the most common mechanisms used in market-based approaches. In an auction, a set of items is offered by an auctioneer in an announcement phase, and the participants can make an offer for these items by submitting bids to the auctioneer. Once all bids are received or a prespecified deadline has passed, the auction is then cleared in the winner determination phase by the auctioneer who decides which items to award and to whom. In robotic applications, the items for sale are typically tasks, roles, or resources. The bid prices reflect the robots' costs or utilities associated with completing a task, satisfying a role, or utilizing a resource.

The simplest kind of auction is a single-item auction in which only one item is offered. In such auctions, each participant submits a bid, and the auctioneer awards the item to the highest bidder. ${ }^{1}$ Alternatively, the auctioneer retains the item if no bid beats the auctioneer's price (called a reserve price). Bids are usually submitted only to the auctioneer; such sealed-bid auctions are in contrast to open-cry auctions where bidders have the benefit of overhearing the other bids as they are made. There are two common approaches to determining the sale price of the auctioned item. In a first-price auction, the sale price is the same as the winning bid; in a Vickrey auction, the sale price is the value of the second-highest bid and is intended to motivate truthful bids from the participants. Some multirobot systems have used Vickrey auctions (e.g., [3]), though the resulting allocations are equivalent to firstprice auctions if the robots are designed to behave truthfully. Wolfstetter provides an excellent introductory survey into single-item auction theory [4].

Combinatorial auctions are more complex: multiple items are offered and each participant can bid on any combination of bundles (i.e., subsets) of these items. This allows the bidder to explicitly express the synergies between items. In the context of the Mars distributed sensing scenario, a bidder can express the positive synergy

${ }^{1}$ We will assume utility maximization here; the case of cost minimization is analogous, with awards going to the lowest cost bidders. between two sites that are close together by bidding only slightly higher for the bundle containing these tasks than for either task individually. To express the negative synergy between two tasks located far from one another, the bid for the bundle would be much higher than the sum of the individual costs of the tasks. In general, there are an exponential number of bundles to consider which makes bid valuation, communication, and auction clearing intractable if all bundles are considered [5].

In between these two extremes are multi-item auctions in which multiple items are offered but the participants can win at most one item apiece. The maximum number of awards per auction may also be limited. Multi-item auctions are a special case of combinatorial auctions where only bundles of cardinality one are considered; bidding and clearing become tractable, but the resulting solutions are generally much less efficient.

\section{Costs, Utilities, and Valuation}

The example scenario in Section I compares robots' suitability for tasks in terms of cost. That is, the auction allocates tasks to the robots with the lowest costs for performing them and the overall goal is to minimize some global cost function. As suggested in Section II-B, in some systems bids are compared based on utilities, in which case the highest bids win auctions and the system attempts to maximize the global utility function. Utilities often encapsulate multiple factors, some representing the benefit or expected quality of task execution and others representing cost estimates. Cost estimates can also include diverse factors such as the time taken to compute solutions and the loss of efficiency caused by transitioning between tasks. As an example of utility, Gerkey and Matarić [6] propose taking the difference of quality and cost to calculate utility, assuming the units of cost and quality are directly comparable. Thus, utility and cost functions that combine multiple factors often require finding a reasonable set of weights between the different components considered.

The process of estimating costs for bid valuation can also be difficult. Though participants in the market may have well-defined cost or utility functions, these functions still rely on having accurate models of the world state and may require computationally expensive operations. For example, the cost to complete the task of driving to a goal site depends on having an accurate map of the environment; however, the robots may be working in an unknown, partially known, or changing environment. When there are multiple goal locations, determining the cost to perform even one task can require solving multiple path planning problems and an instance of the traveling salesman problem (TSP), the latter being $\mathcal{N} \mathcal{P}$-hard. Thus, heuristics and approximation algorithms are commonly used, implying that bid prices may not always be entirely accurate. Inaccurate bids can result in tasks not being awarded to the robots best able to complete them. In this case reauctioning tasks can often improve solution quality. 


\section{The Range of Coordination Approaches}

The goal in virtually all robotic application domains is to generate optimal solutions in a timely manner. Unfortunately, many multirobot coordination problems are $\mathcal{N} \mathcal{P}$-hard. The challenges are compounded by team considerations that include operation in dynamic and uncertain environments, inconsistent information, unreliable and limited communication, interaction with humans, and various system and component failures. A spectrum of coordination approaches has emerged to negotiate these demands.

At one end of the spectrum, fully centralized approaches employ a single agent to coordinate the entire team. In theory, this agent can produce optimal solutions by gathering all relevant information and planning for the entire team. In reality, fully centralized approaches are rarely tractable for large teams, can suffer from a single point of failure, have high communication demands, and are usually sluggish to respond to local changes. Thus, centralized approaches are most suited for applications involving small teams and static environments or easily available global information.

At the other end of the spectrum, in fully distributed systems, robots rely solely on local knowledge. Such approaches are typically very fast, flexible to change, and robust to failures, but can produce highly suboptimal solutions, since good local solutions may not necessarily aggregate to a good global solution. Applications where large teams carry out relatively simple tasks with no strict requirements for efficiency are best served by fully distributed coordination schemes.

A vast majority of coordination approaches have elements that are centralized and distributed and thus reside in the middle of the spectrum. Market-based approaches fall into this hybrid category, and, in some instances, they can opportunistically adapt to dynamic conditions to produce more centralized or more distributed solutions. Market mechanisms can distribute much of the planning and execution over the team and thereby retain the benefits of distributed approaches, including robustness, flexibility, and speed [7], [8]. Auctions quickly and concisely assemble team information in a single location to make decisions about distributing resources; in some cases they provide guarantees of solution quality [5], [9]. Market-based approaches may also incorporate methods of opportunistically coordinating subteams in a centralized manner [10], [11]. Nevertheless, market-based approaches are not without their weaknesses. In domains where fully centralized approaches are feasible, marketbased approaches can be more complex to implement and produce poorer solutions. In domains where fully distributed approaches suffice, market approaches can be unnecessarily complex in design and have greater communication and computation requirements.

The sections that follow discuss market-based multirobot coordination in greater detail along the dimensions mentioned in the introduction. Each section introduces the topic and its challenges, defines the goals and appropriate evaluation metrics, reviews the relevant literature, and identifies remaining research challenges.

\section{PLANNING}

In multirobot teams, planning can be required to coordinate robots to accomplish the team mission. Unfortunately, optimal planning problems for multirobot systems are typically $\mathcal{N} \mathcal{P}$-hard [12]. The challenge then is to have tractable planning that produces efficient solutions. Market-based approaches manage this by distribut-

\section{Auctions quickly and concisely assemble team information in a single location to make decisions about distributing resources; in some cases they provide guarantees of solution quality.}

ing planning over the entire team to produce solutions quickly. When required or when resources permit, markets can behave in a more centralized fashion and plan over larger portions of the team to improve solution quality. Here, we consider different layers at which planning arises in a multirobot system and how these planning problems are handled by various market-based approaches.

\section{A. Related Work}

1) Planning and Task Allocation: Task allocation is the problem of feasibly assigning a set of tasks to a team in a way that optimizes a global objective function. Many special cases of task allocation appear frequently in the literature; here, we offer a general and formal definition that allows us to discuss and compare them.

Definition 1: Given a set of robots $R$, let $\mathcal{R}:=2^{R}$ be the set of all possible robot subteams. An allocation of a set $T$ of tasks to $R$ is a function, $A: T \rightarrow \mathcal{R}$, mapping each task to a subset of robots responsible for completing it. Equivalently, $\mathcal{R}^{T}$ is the set of all possible allocations of the tasks $T$ to the team of robots $R$. Let $T_{r}(A)$ be the set of tasks allocated to subteam $r$ in allocation $A$.

Definition 2: The Multirobot Task Allocation Problem: Given a set of tasks $T$, a set of robots $R$, and a cost function for each subset of robots $r \in \mathcal{R}$ specifying the cost of completing each subset of tasks, $c_{r}: 2^{T} \rightarrow \mathbb{R}^{+} \cup\{\infty\}$, find the allocation $A^{*} \in \mathcal{R}^{T}$ that minimizes a global objective function $C: \mathcal{R}^{T} \rightarrow \mathbb{R}^{+} \cup\{\infty\}$. 
Gerkey and Matarić [6] provide a taxonomy for some variants of the task allocation problem, distinguishing between: single-task (ST) and multitask (MT) robots; single-robot (SR) and multirobot (MR) tasks; and instantaneous (IA) and time-extended (TA) assignment. In instantaneous assignment robots do not plan for future allocations and are only concerned with the one task they are carrying out at the moment or for which they are bidding. In time-extended assignment robots have more information and can come up with longer term plans involving task sequences or schedules. Definition 2 encompasses each of the types of task allocation in the taxonomy, but in general describes TA task allocation. IA allocation can be represented as a special case where all cost functions map to infinity for any subsets of tasks with cardinality greater than one. Further, if we allow the sets of tasks $T$ and robots $R$ to be time dependent (i.e., $T(t)$, $R(t)$ ) and require the objective function be minimized at every instant of time or over the entire history, then the definition also covers online and dynamic domains where tasks and robots may be added or removed over time (see Section VI). This definition also implies that task allocation is $\mathcal{N} \mathcal{P}$-hard in general, as the multidepot traveling salesman problem is a special case [12].

Market-based approaches distribute planning required for task allocation through the auction process: each robot or group of robots locally plans the achievement of the offered tasks, computes its costs, and encapsulates the costs in its bids. This process is illustrated in the introduction of this paper for a distributed sensing task on Mars: each robot determined its own cost of visiting different sites. Most existing market-based approaches fall into the SR-ST category in the task allocation taxonomy. Several assume instantaneous assignment (IA) [7], [13]-[15], while others allow for time-extended assignment (TA), introducing an additional layer of planning whereby robots sequence [9], [16]-[20] or schedule [21]-[23] a list of tasks and can therefore explicitly reason about the dependencies between multiple tasks and upcoming commitments. More recently, market-based systems have addressed the allocation of multiple-robot tasks (MR-ST) [24], [25], including human-robot tasks [26].

Market-based mechanisms for task allocation can also be differentiated as centralized or distributed. Centralized mechanisms have the ability to find optimal solutions (e.g., through combinatorial auctions [5], [16]) or provide bounds on solution quality [9], but in general can require an exponential amount of computation and communication [5]. Distributed mechanisms [17], [18] act as anytime algorithms and require less computation and communication resources, but are not guaranteed to find optimal solutions and have no known approximation bounds. TraderBots [17] attempts to find a balance between these two approaches by opportunistically allowing "pockets" of centralized optimization to emerge within subgroups of the team when resources permit. In our distributed sensing example, for instance, the team might begin with a suboptimal allocation of sites, perhaps caused by an inaccurate map of the environment resulting in inaccurate bids. At some point during execution (perhaps when map information is more accurate), a robot might find a better distribution of sites for some subset of its teammates. The robot's motivation for group optimization is that it can pocket the cost difference as profit by winning the tasks from the original holders and subcontracting them to the new holders. Simultaneously, this results in a better team solution. Solution quality and scalability aspects of these different approaches are discussed in more detail in Sections IV and V, respectively.

Related problems of allocating constrained subtasks and roles can have additional planning requirements.

a) Allocating Constrained Subtasks: In many domains, tasks are temporally constrained with respect to one another. They may be partially ordered or may need to start or finish within a common time frame. For instance, consistency may be important in our Mars distributed sensing task, so we might require that samples from particular sites be collected at the same time. In the case of partially ordered tasks, one can use a central allocator to auction only those tasks whose predecessors have been completed [27]. Alternatively, during assignment, robots can incorporate the cost of meeting constraints into their bids [28]. In terms of Definition 2, a violation of constraints can be modeled as infinite values for local cost functions or the global objective function. Constraints can add another dimension to the bid valuation and auction clearing processes and may thus increase computation requirements. Often, robots must also coordinate during execution to reschedule and accommodate team and task changes that have occurred since the initial allocation [21], [22]. In these cases, robots must be able to determine when and how the rescheduling should occur.

b) Allocating Roles and Instantaneous Assignment: In team games one usually assigns positions such as "primary offense" or "supporting defense" instead of tasks such as "shoot the ball" or "capture a rebound." These positions can be classified as roles. More generally a role defines a collection of related actions or behaviors. Indeed, in many domains it is more natural to think of teammates playing roles than completing distinct tasks. In market-based approaches, role allocation can use the same auction-bidaward protocol as task allocation. However, robots can usually take on only one role at any given time (SR-ST-IA or MR-ST-IA) and generate bids by evaluating a fitness function that reflects how well its current state matches the requirements of the role. Once allocated, a robot locally plans the execution of actions and behaviors specified by its role. Market-based role allocation has been demonstrated in robot soccer [13], [15] and treasure hunt [29] domains.

Instantaneous assignment (IA) also arises in cases where the tasks being allocated are short-term partial actions that bring the team goal closer to being realized. 
Examples of instantaneous assignment include allocating push actions in a box-pushing application [7] and assigning waypoint locations (that do not necessarily have to be reached) in an exploration scenario [14].

2) Planning and Task Decomposition: Although many approaches to task allocation assume that a list of primitive

\section{Market-based approaches distribute planning required for task allocation through the auction process: each robot or group of robots locally plans the achievement of the offered tasks, computes its costs, and encapsulates the costs in its bids.}

or simple tasks is input to the system, a complex mission is often more naturally described at a higher level of abstraction. For example, scientists desiring data about Mars may only be concerned with the general regions from which data is collected and not the precise sites. As illustrated in Fig. 1, a mission might be phrased as "capture images that collectively show $50 \%$ of crater regions A, B, C, and D." In these cases, multirobot systems must also decompose a mission into subtasks, often making use of well-known planners [27], [30] or domain-specific decomposition algorithms [31].

There are two common approaches to this planning problem. In the decompose-then-allocate method, a single agent recursively decomposes the task into simple subtasks which are allocated to the team [30]. In the distributed sensing scenario, this amounts to finding a fixed set of observation sites for all crater regions and then allocating these sites to the team. In the allocate-then-decompose method, complex tasks are first allocated to robots then each robot locally decomposes its awarded tasks [27]. This corresponds to assigning entire crater regions to robots and letting each robot choose the sites. It is also possible to include instances of both techniques [21], [27].

By decoupling the decomposition and allocation problems, these approaches do not consider the complete solution space and may find highly inefficient solutions. In general, one cannot decompose a task optimally without knowing which robots will execute the subtasks, nor can one allocate tasks efficiently without knowing how they will be decomposed. One solution is to simultaneously work on both problems by generalizing tasks to task trees and trading these explicitly on the market [31]. By incorporating bids at multiple levels of abstraction, both the costs of allocations and plans can be compared in a single auction mechanism. Experiments in complex task domains demonstrate that using task tree auctions can improve solution quality over two-stage approaches [31].

3) Planning and Task Execution: Many missions including our example distributed-sensing mission on Mars consist of single-robot (SR) tasks that can be completed independently by individual robots. Such missions can usually be achieved by a loosely coordinated team in which robots coordinate during task decomposition and allocation but not during execution. Thus, in these domains, planning the execution of tasks can be done at an individual level without consideration of teammates' actions. The exception is when robots unexpectedly interfere with each other during execution. Azarm and Schmidt [32] address collision avoidance of independent robots during execution using market-based techniques.

However, another class of problems which includes manipulating large objects (e.g., beams to construct a structure) and moving in formation (e.g., to safely travel between sites of interest) cannot easily be decomposed into independent subtasks. These domains require tightly coordinated teams in which members continuously coordinate throughout execution. Tight coordination is extremely challenging: teams must essentially solve a tightly coupled multirobot planning problem, but cannot easily take advantage of the distributed planning and execution that make loose coordination tractable.

Market-based approaches are not often used to plan the interactions of tightly coordinating teammates. In practice, these interactions are simple in many domains and can be achieved with reactive or behavior-based approaches that forgo planning and are less expensive in terms of design, computation, and communication. For instance, Simmons et al. [33] use a team to perform largescale construction. They propose a market-based approach to select robots for manipulation tasks that require tight coordination, but execute the tight coordination using a reactive approach.

However, some domains greatly benefit from and even require advanced planning of the interactions between robots. For example, we may want our Mars rovers to always stay in communication contact with a base station; doing so requires that they tightly coordinate over large distances and plan paths to sites with each others' actions in mind. Reactive and behavior-based approaches cannot provide such extensive planning; instead, market-based approaches have recently been developed to address these domains. The idea is to exploit small pockets of centralized planning by having robots buy and sell tightly coupled joint plans over the market rather than independent tasks [11]. These approaches have higher communication and computational demands than most market-based approaches designed for loosely coordinated teams. 


\section{B. Future Challenges}

Multirobot systems typically must incorporate multiple types of planning for different aspects of the problems they address. Market-based approaches are currently capable of several types of planning, but several challenges remain. First, there has been limited work in domains with many complex constraints between tasks and domains requiring tight coordination. Second, efficient replanning is crucial to working in uncertain environments and relates closely to issues raised in Section VI. Task reallocation can be achieved by peer-to-peer trading and some progress has been made in redecomposing complex tasks in marketbased systems [31], but significant work remains in replanning for tightly coordinated teams. A third important and relevant area of research (for which some initial work has been done [29]) is understanding the formation of subteams and enabling their positive interaction using market-based methods. Finally, market-based approaches need better strategies for making use of multirobot planners and providing alternatives to combinatorial auctions for vetting complex plans in the market.

\section{QUALITY OF SOLUTION}

One of the greatest strengths of a market is its ability to utilize the local information and preferences of its participants and arrive at an efficient solution given limited resources. As discussed in Section III, a fundamental optimization problem encountered in marketbased multirobot systems is the task allocation problem. Since task allocation is $\mathcal{N} \mathcal{P}$-hard system designers face the challenge of choosing market mechanisms that result in the most efficient solutions within a reasonable amount of time. Various global cost objectives ( $C$ in Definition 2) appear in market-based systems depending on the application. Most common are minimizing the sum of individual robots' costs $\left(C(A)=\sum_{r \in \mathcal{R}} c_{r}\left(T_{r}(A)\right)\right)[16]-[18]$, [34], [35] or minimizing the maximum individual cost (makespan, $C(A)=\max _{r \in \mathcal{R}} c_{r}\left(T_{r}(A)\right)$ ) [19]; although others are possible (e.g., minimizing the average overall time to complete each task [36]). In the Mars distributed sensing example, these global objectives correspond to finding the allocation of sites to robots that results in the least amount of fuel expended (sum of costs) or the task being done in the least amount of time (makespan). While it has been demonstrated that inaccuracies in cost models can affect the quality of solutions obtained [37], the results presented in this section are all developed under the assumption that the robots' cost or utility estimates are accurate.

\section{A. Related Work}

As described in Section III, we distinguish between instantaneous (IA) and time-extended (TA) task allocation. The IA model often arises in cases where tasks require time-indefinite exclusive commitments by robots (e.g., roles [13], [15]) or when the tasks being assigned are shortlived partial actions that bring the team goal closer to being realized [7], [14]. However, sometimes IA approaches are used for simplicity in lieu of a TA approach; they are easier to implement and do not need computationally expensive task sequencing algorithms [6], [24], [30], [38]. In these systems, if there are more tasks than robots, the remaining tasks can be allocated once robots complete their previous assignments. Ignoring dependencies between tasks by using an IA approach should hypothetically result in inferior solution quality, but we are not aware of any explicit comparative study. When IA allocation is appropriate, it has been demonstrated that optimal allocation is possible if there are more robots than tasks, although several existing systems use a 2-approximate greedy solution [6]. Additionally, performance guarantees are not always equivalent for cost- and utility-based systems; the greedy algorithm for the metric online variant of the IA task allocation problem is 3-competitive for utility maximization [6], [39] but scales exponentially with the number of robots for cost minimization [39].

TA sequencing approaches have additional planning and scheduling requirements, but, when appropriate, model the problem more accurately and should produce better results. For example, a combinatorial auction can theoretically result in an optimal allocation if the robots compute and submit bids on all possible combinations of tasks (of which there are an exponential number) [5]. In practice, performance guarantees are sacrificed in order to reduce the computation and communication requirements by considering only a relatively small number of task bundles [16], [34], [40], [41] (details in Section V).

A simpler centralized mechanism is one in which single tasks are iteratively allocated in multiple auctions until all tasks are assigned. In general, these types of auctions are not guaranteed to find the optimal solution; however, because they require less computation and communication than combinatorial auctions and are easier to implement, they are more prevalent in the literature [18]-[20], [27], [30], [35]. Tovey et al. [36] suggest a hillclimbing heuristic for generating bidding rules for singletask auctions with various global objective functions. This method gives some justification for the typically utilized bidding rules: for example, when globally minimizing total cost, bidders should base their bids on the marginal costs of the offered tasks [16]-[18], [34], [35]; for makespan minimization, load balancing can be better achieved if participants instead bid based on their total costs [19]. Experimentally, Tovey et al. found that the bidding rule derived for each of three objective functions (total cost, makespan, and average latency) results in better solutions than the rules derived for the other objectives. By modeling multirobot problems as vehicle routing problems [45]-[47], Lagoudakis et al. [9] provide a set of approximation bounds for the same rules and objective 
Table 1 Summary of Solution Quality Results

\begin{tabular}{|l||l|l|}
\hline \multicolumn{1}{|c||}{ Approach } & \multicolumn{1}{|c|}{ Theoretical guarantees } & \multicolumn{1}{c|}{ Experimental results } \\
\hline \hline $\begin{array}{l}\text { Combinatorial auctions } \\
\text { [16], [34] }\end{array}$ & Optimal (if all bundles are considered) [5] & $\begin{array}{l}\text { Good solutions with limited number of task bun- } \\
\text { dles [16]. [34], [40], [41] }\end{array}$ \\
\hline $\begin{array}{l}\text { Central single task iterated } \\
\text { auctions [9], [36] }\end{array}$ & $\begin{array}{l}\text { Approximation bounds for 18 cases (3 objective } \\
\text { Close to optimal results when using the appropri- } \\
\text { ate bidding rules [36] }\end{array}$ \\
\hline $\begin{array}{l}\text { Central instantaneous as- } \\
\text { signment (IA) [7], [13] }\end{array}$ & $\begin{array}{l}\text { Optimal 6 bidding rules) [9] } \\
\text { rithm is a 2-approximation; greedy algorithm for } \\
\text { online version is 3-competitive [6] }\end{array}$ & \\
\hline $\begin{array}{l}\text { Peer-to-peer trading [17], } \\
\text { [18], [19], [20], [35] }\end{array}$ & $\begin{array}{l}\text { Optimal solution possible in a finite number of } \\
\text { trades with a sufficiently expressive set of contract } \\
\text { types [42] }\end{array}$ & $\begin{array}{l}\text { In a limited number of rounds, a combination of } \\
\text { single- and multi-task trades outperforms all other } \\
\text { combinations of single-task, multi-task, swap, and } \\
\text { multi-party contracts [12]; allowing non-individual } \\
\text { rational trades can lead to better solutions [43] }\end{array}$ \\
\hline $\begin{array}{l}\text { Central multi-task auctions } \\
\text { followed by peer-to-peer } \\
\text { trading [44] }\end{array}$ & $\begin{array}{l}\text { Increasing the maximum number of tasks awarded } \\
\text { per multi-task auction results in poorer solution } \\
\text { quality [44] }\end{array}$ \\
\hline
\end{tabular}

functions. They prove that bids based on individual marginal costs when applied to a sum-of-costs objective results in a 2-approximation, while bids based on individual total costs applied to a makespan objective yields an approximation algorithm that scales linearly with the number of robots (which is a worst case result for any makespan algorithm).

Task reallocation is also possible by introducing peerto-peer auctions [17], [18], [22], [35], [44]. In this case, there is some initial allocation, and any robot on the team is capable of holding auctions in order to reallocate tasks to robots that are better suited to perform them. In static environments, distributed trading can improve inefficient initial allocations resulting from the use of faster but suboptimal mechanisms. In unknown or partially known environments where costs constantly change as new observations are made, initial solutions may no longer maintain optimality guarantees or even be reasonably efficient; in such a case the use of peer-to-peer auctions can be used to repair undesirable allocations. Peer-to-peer trading can be viewed as a local search and thus is subject to local optima. Sandholm proves that by using a sufficiently expressive set of contract types (single-task, multitask, swap, and multiparty), the global minimum can be reached in a finite (but possibly large) number of steps [42], while experiments by Andersson and Sandholm demonstrate that more practical systems that include just single- and multitask contracts (e.g., [41]) find the most efficient solutions given a limited number of rounds [12]. Another interesting result by Vidal [43] shows that by not requiring agents to be purely selfish (i.e., some agents may be worse off after some trades) the local search algorithm can circumvent some local optima and in the long run find better solutions. Dias et al. [44] look at how to initialize the team allocation by holding central greedy multitask auctions (multiple tasks can be awarded in each but at most one task is awarded per robot per auction) before distributed trading begins. They find that increasing the number of tasks awarded per auction can have a negative effect on the resulting solution quality but requires less time (fewer auctions are held) to find a solution. Table 1 gives a summary of the results presented in this section.

\section{B. Future Challenges}

While some theoretical guarantees for simple auctions are known, future work should address the more complex mechanisms that are present in implemented systems which can include online, multitask, peer-to-peer, simultaneous, and overlapping auctions as well as task and scheduling constraints. Additionally, solution quality depends on accurate cost and utility measures which may be very challenging to aquire. Although some progress has been made in methods for learning [23] and improving [37] these estimates, further work is required.

\section{SCALABILITY}

Scalability is an important feature for any multirobot coordination approach. In general, a system is scalable if it can operate effectively even as the number of inputs or the size of inputs increases arbitrarily. The scalability of a multirobot coordination approach is typically evaluated by its ability to produce efficient solutions as the team size or the task complexity increase. For example, in the Mars distributed sensing scenario, a scalable coordination approach will continue to produce efficient task allocations as the number of robots in the team and the number of sensing tasks assigned to the team increase. Scalability in some market-based approaches may be limited by the computation and communication needs that arise from increasing auction frequency, bid complexity, and planning demands. However, market-based approaches can scale well in applications where the team mission can be decomposed into tasks that can be independently carried out by small subteams. A scalability comparison of marketbased, behavior-based, and centralized approaches is presented by Dias and Stentz [17] on a distributed sensing task in which the goal is to visit the last observation site in as little time as possible (i.e., a makespan objective). Simulation experiments demonstrate that the 
market can provide significantly higher quality solutions than behavior-based approaches while using significantly less computation time than centralized approaches.

\section{A. Related Work}

1) Computation and Communication Considerations: Section IV referred to tradeoffs between scalability and solution quality in market-based systems. Here, we elaborate on some of these tradeoffs with respect to common auction types. Single-item auctions are usually computationally feasible and light on communication, but they produce suboptimal solutions. Combinatorial auctions can produce optimal solutions, but require an exponential amount of computation and communication. Multi-item auctions are also computationally manageable, but produce inferior solutions as compared to single-item and combinatorial auctions. Their advantage is that more items are awarded per auction so items can be allocated quickly, since less auctions are required. When considering these tradeoffs, it is also important to consider the problem domain: for highly uncertain or dynamic environments, it may not be worth spending the time to compute an optimal solution if that solution will constantly be changing as more information is gathered; or, if there are hard real-time constraints, there may not be enough time to compute an exact solution.

Table 2 summarizes the time complexities of the important phases of several auction types. For each protocol, the table lists the maximum number of bid valuations, the computation times of the best-known auction clearing algorithms, and the number of auctions required to allocate all items to the team (if the objective is to offload all items from the initial auctioneer - this may not be the aim in peer-to-peer auctions as the auctioneer may retain some items or some reserve prices may not be met). Table 3 similarly gives a summary of communication costs. Of particular interest in these tables are the exponential expressions for combinatorial auctions in the areas of bid valuation, winner determination, and bid submission. As discussed previously, in order to make combinatorial auctions scalable, the common approach is to limit the

Table 2 Comparison of Time Complexities of Various Auction Types. $n$ is the Number of Items, $r$ is the Number of Bidders, $b$ is the Number of Bids, and $m \leq r$ is the Maximum Number of Awards per Auction (for Multi-Item Auctions). $v$ and $v$ Represent the (Domain-Dependent) Amount of Time Required to Perform a Valuation for a Single Item $(v)$ or Set of Items $(V)$

\begin{tabular}{|c||c|c|c|}
\hline Auction type & $\begin{array}{c}\text { Bid } \\
\text { valuation }\end{array}$ & $\begin{array}{c}\text { Winner } \\
\text { determination }\end{array}$ & $\begin{array}{c}\text { Number of } \\
\text { auctions }\end{array}$ \\
\hline \hline Single-item & $v$ & $O(r)$ & $n$ \\
\hline $\begin{array}{c}\text { Multi-item } \\
\text { (greedy) }\end{array}$ & $O(n \cdot v)$ & $O(n \cdot r \cdot m)$ & $\lceil n / m\rceil$ \\
\hline $\begin{array}{c}\text { Multi-item } \\
\text { (optimal) }\end{array}$ & $O(n \cdot v)$ & $O\left(r \cdot n^{2}\right)[6]$ & $\lceil n / m\rceil$ \\
\hline Combinatorial & $O\left(2^{n} \cdot V\right)$ & $O\left((b+n)^{n}\right)[5]$ & 1 \\
\hline
\end{tabular}

Table 3 Comparison of Communication Complexities of Various Auction Types. Notation is the Same as in Table 2

\begin{tabular}{|c||c|c|c|c|}
\hline Auction type & $\begin{array}{c}\text { Auction } \\
\text { call }\end{array}$ & $\begin{array}{c}\text { Bid } \\
\text { submission }\end{array}$ & Award & $\begin{array}{c}\text { Award } \\
(+ \text { losers })\end{array}$ \\
\hline \hline Single-item & $O(r)$ & $O(r)$ & $O(1)$ & $O(r)$ \\
\hline Multi-item & $O(r \cdot n)$ & $O(r \cdot n)$ & $O(m)$ & $O(r)$ \\
\hline Combinatorial & $O(r \cdot n)$ & $O\left(r \cdot 2^{n}\right)$ & $O(n)$ & $O(r+n)$ \\
\hline
\end{tabular}

number of bundles that are considered during bid valuation. This in turn reduces the number of bids that need to be communicated and also allows the auction to be cleared quickly in practice. Indeed, Sandholm's optimal clearing algorithm, САВОВ [5], relies on a sparse bid set in order to find solutions quickly (although the resulting allocation is still likely to be suboptimal given that not all item bundles are considered). The number of bundles can be reduced either on the auctioneer's side by offering only certain combinations of items [40], or on the bidders' side by using heuristic item clustering algorithms [16], [41] or by ignoring bundles greater than a certain size [16], [34]. Berhault et al. [16] compare four clustering algorithms for goal point tasks and find that one based on repeated graph cuts outperforms a nearest-neighbor algorithm and two algorithms based on limiting cluster size.

Bid valuation itself may be computationally expensive. That is, the expressions $v$ and $V$ in Table 2 may in reality represent the running time of algorithms that must solve a difficult or even $\mathcal{N} \mathcal{P}$-hard problem in order to estimate costs [16]-[19], [35]. Additionally, expensive task decompositions may be required at the bidding stage [31]. Heuristics and approximation algorithms can help deal with the $\mathcal{N} \mathcal{P}$-hard problems (e.g., TSP approximation algorithms for task sequencing [10]), although when there are many items to consider simultaneously - either from auctions offering many items [16]-[18], [22], [31], [34] or from multiple robots holding auctions simultaneously [17], [31] — bidders can still be overburdened with valuation problems. As a result, system designers must ensure that bidders are able to meet auction deadlines and do not tax their processors to the point of compromising real-time requirements.

2) Opportunistic Centralization: Opportunistic centralization [11], [41] is a scalable way of incorporating the benefits of centralized planning into large market-based systems. In such approaches, centralized resource or task allocation can be done over smaller subsets of tasks and team members as and when computational resources permit. This is a relatively new area in market-based coordination; consequently, there are no theoretical formulations and only a few experimental results.

\section{B. Future Challenges}

While much is known theoretically about the scalability of various auction mechanisms, market-based 
approaches have yet to be implemented on teams of more than a few robots. Further challenges exist in improving opportunistically centralized approaches' means of selecting task clusters and team members to reduce unnecessary computation. The challenge of dealing with limited computation when faced with an excess of solicited bid valuations is also largely unaddressed.

\section{DYNAMIC EVENTS AND ENVIRONMENTS}

Operations in dynamic and uncertain environments pose a variety of challenges to team coordination: ensuring graceful degradation of solution quality with failures, enabling team functionality despite imperfect and uncertain information, maintaining effective response speed to dynamic events, and accommodating evolving conditions and constraints. Benchmarking the robustness of a coordination approach requires evaluating the diversity of failures the team can accommodate, the required quantity and certainty in information available to the team, the team's response speed to dynamic events, the fluidity of the team, and the overall solution quality produced by the team in the face of dynamic events. In this section we examine the different ways in which market-based multirobot coordination approaches to date deal with these conditions.

\section{A. Related Work}

1) Robustness and Fluidity: Robotic systems are often complex and their physical interactions with the environment make them highly prone to failures. Thus, a successful coordination approach must gracefully degrade solution quality when failures occur. For example, in the Mars distributed sensing scenario, it is likely that one or more of the rovers will suffer some form of failure. A rover can wander outside the range of communication from other rovers, damage a scientific instrument when performing a sensing task, or become completely disabled due to a rock slide. A successful coordination approach will allow the team to accomplish the mission despite these failures. Three principal categories of faults that coordination approaches must consider are communication failures, partial malfunctions, and robot death [8]. A variety of strategies are employed by market-based approaches to handle these failures.

Teams can often perform more effectively if teammates can communicate [20]. However, communication failures occur in a variety of domains and range from occasional loss of messages to loss of all communication. In the TraderBots approach, if the team is informed of a common task, consequent disruptions in communication are gracefully handled by using opportunistic auctioning solely for improving solution quality [8], [20]. As with communication disruptions, partial malfunctions limit a robot's capability but retain the robot's planning ability. TraderBots employs active reasoning about failed resources to allow robots to reallocate tasks that they can no longer complete due to malfunctions [8]. Similarly, MURDOCH relies on monitoring progress in short-duration tasks to detect and respond to faults [7]. In the case of robot death, the affected robot cannot aid in the recovery process. However, robots can monitor progress or heartbeats of teammates and reauction tasks previously assigned to the dead robot [7], [8]. A further improvement is to allow repair of malfunctioning robots and enable their return to the team. In order to accomplish this, a coordination approach must accommodate both the exit of malfunctioning robots and the entrance of the repaired robots. Bererton et al. demonstrate reasoning about assisting malfunctioning robots and towing of disabled robots to a base station for repair [48], while Dias et al. [8], [10] and Gerkey and Matarić [7] demonstrate reentry of repaired robots (or entry of new robots) to the team. When dealing with dynamic and uncertain conditions, coordination approaches must wrestle with several tradeoffs. For example, if detecting robot death relies on monitoring a heartbeat, a robot is presumed dead if its heartbeat is not received by its teammates within a predetermined interval. However, if this interval is too large, the time to detect and respond to a failure is increased and the solution quality is thus degraded. Instead, if the interval is too short, solution quality can still degrade due to false positives that can arise if the robot temporarily drops out of communication range. Understanding these tradeoffs and their implications remains an open problem.

2) Online Tasks: In many dynamic application domains, the demands on the robotic system can change during operation. Operators of a multirobot system may submit new tasks or alter or cancel existing tasks [10]. Alternatively, robots may generate new tasks during execution as they observe new information about their surroundings. In the Mars scenario, a scientist may choose to add new sites to explore or eliminate existing sites while reviewing incoming data, or the robots themselves may have the capacity to make such decisions. Market-based approaches can often seamlessly incorporate online tasks by auctioning new tasks as they are introduced by an operator [17], [26] or as they become available due to the completion of preceding tasks [7], [14], [27], [30]. In some cases new tasks can be generated by the robots themselves and inserted into their plans to be executed or subsequently traded [10], [20], [24].

3) Uncertainty: Most real-world multirobot applications require operation with only partial or changing information about the environment, the team, or the task. Fortunately, market-based approaches have few a priori information requirements and can accommodate new information through frequent auctioning of tasks and 
resources. For instance, in the Mars example, it is likely that the rovers will not have access to a complete detailed map of their environment. The TraderBots approach demonstrates that robots can execute tasks with no a priori map information and dynamically reallocate tasks when new map information is gathered [10], [20].

\section{B. Future Challenges}

While much can be done to improve the operation of market-based approaches in dynamic environments, a few key challenges are paramount. Effective information sharing among team members in market-based approaches is one necessary area of research. If a robot discovers a task is expensive because of new environmental information it has gathered, it can potentially allocate the task to another robot that does not have that information. This robot will then try to execute the task until it, too, perceives the new information; then it tries to allocate the task to another robot. This can continue until all robots have attempted to perform the task, causing tremendous inefficiency.

Characterizing the ability of both the individual robots and the team to respond quickly to dynamic conditions using market-based coordination approaches is another important challenge. The authors are not aware of any study of individual or team response speed for any marketbased multirobot coordination approach. Other challenges for improving robustness are developing more sophisticated methods for cooperative handling of partial malfunctions and repairs, evaluating robustness to a variety of failures, incorporating contract breaches with appropriate penalties, and incorporating sliding autonomy into marketbased approaches to allow robots to request assistance when appropriate.

\section{HETEROGENEOUS TEAMS}

A team is heterogeneous if not all of its members are equally capable of performing all the tasks (e.g. , because of hardware or software differences) or if its members play different roles (e.g., in team games where robots play different positions). In contrast, the members of a homogeneous team have identical skills or are generalists that can perform all necessary tasks. Heterogeneity is highly advantageous for several reasons. First, complex missions often have many different functional requirements and can be achieved more effectively by a team of specialists rather than by a team of generalists that perhaps cannot perform any single function very well. For example, in our Mars scenario we may want high-resolution images taken, rock samples collected, and core samples taken from the ground. A complex mission such as this is often better achieved with robots that specialize in particular tasks: some robots can take samples from both rocks and the ground while others only capture images. Second, it is often more practical to design robots that specialize in only a small set of skills than to design robots that are capable of all skills. Indeed, in many domains, it may be infeasible to construct robots that can do everything, for example because of limitations in budget, form factor, or on-board power. Third, by being able to coordinate heterogeneous teams, we can reuse robots across multiple applications. Although heterogeneous team coordination is challenging, a successful approach should accommodate any team composition.

\section{A. Related Work}

In a heterogeneous team, robots have different abilities to perform different tasks. Task and role allocation in heterogeneous teams becomes challenging because it requires reasoning about and comparing different robots' capabilities. Market-based approaches are well suited to meet this challenge because auctions can simplify the problem of reasoning about team skills. When a task or role is auctioned, each robot's bid encapsulates its ability to complete the task in terms of resource usage or even the opportunity cost of forgoing other tasks [23]. Additionally, robots can abstain from bidding on tasks for which they do not have sufficient resources, thereby reducing the computational burden for both bidder and auctioneer [7], [40]. The auctioneer can award roles or tasks to team members according to the best bid, without requiring knowledge of individual capabilities. Thus, market-based approaches only require each team member to recognize its own skills and resources but not those of teammates. However, auctions introduce a new difficulty: it is not always clear how to compute and compare the cost of performing a task between different types of robots who may perform the task in very different ways. One idea is to allow robots to swap tasks directly whenever such a

\section{While many multirobot coordination approaches have been proposed by the research community, market-based approaches in particular have been proven effective.}

trade results in a mutually beneficial outcome [18]. This circumvents the pricing problem but also severely restricts the number of possible solutions.

Market-based coordination of heterogeneous teams has been demonstrated on physical robots in several applications: in automated assembly using three robots with very different physical configurations and capabilities [33]; in box-pushing, where resource-addressed messaging allows robots to determine in which auctions they should 
participate [17]; and in treasure hunt, with an auctioning scheme in combination with predefined team strategies (known as plays) to allow two very different types of robots to operate as a cohesive team [26]. In simulation, marketcoordinated robots with different science instruments characterize multiple rock types in a space application similar to our Mars scenario [17], and market-based role allocation mechanisms have appeared in heterogeneous robot soccer teams [13], [49].

\section{B. Future Challenges}

Ultimately, coordination approaches must accommodate three levels of heterogeneity: heterogeneous robot teams, human-robot teams, and highly heterogeneous teams of humans, robots, and other agents. Future research challenges include modeling human preferences using appropriate reward functions, developing techniques for consistently computing different robots' costs for completing tasks, enabling pickup teams, i.e., dynamically formed heterogeneous teams where little may be known $a$ priori about the task, the robots, or the environments [29], and addressing the challenges of human-robot teams where tasks are understandable to humans and robots and both participate in task allocation and execution [29].

\section{CONCLUSION AND FUTURE DIRECTIONS}

The vision that drives research in multirobot systems is that teams of robots will inevitably be an integral part of our future. To realize this vision, robots must be capable of executing complex tasks as a team. While many multirobot coordination approaches have been proposed by the research community, market-based approaches in particular have been proven effective; their resulting increase in popularity over the past few years warrants a survey of the field. We address this need by providing the first survey of the state of the art in market-based multirobot coordination approaches with three contributions to the multirobot literature: a tutorial on market-based multirobot coordination approaches, a review and analysis of the relevant literature, and a discussion of remaining challenges in this research area. A more comprehensive version of this paper is presented as a technical report by Kalra et al. [50].

The existing work in market-based multirobot coordination ranges from theoretical formulations to conceptual design frameworks to implementations in simulation and on physical robot teams. The chosen application domains span a wide range and include distributed sensing, mapping, exploration, surveillance, perimeter sweeping, assembly, box-pushing, reconnaissance, soccer, and treasure hunt. However, this is still a relatively new area of research, and hence many research challenges still remain. Here, we discuss some of the overall challenges in the field.

A first important need is a clear conceptual understanding of market-based coordination approaches. Much discussion is needed to further our understanding of how components such as cost and reward functions, bidding strategies, and auction clearing mechanisms can be designed, implemented, and used effectively in different multirobot application domains. Understanding the tradeoff between solution quality and scalability when designing and implementing coordination mechanisms is also important. Additionally, much work still remains in defining a relevant set of benchmarks for effective comparison of different coordination approaches. Some of the challenges in providing a comparative framework for coordination approaches are explored by Gerkey and Matarić [6] who provide an initial framework for evaluating task allocation schemes in terms of complexity and optimality, showing that market-based methods perform favorably in terms of computation and communication requirements. Dias and Stentz [17] compare a centralized optimal approach, a distributed behavioral approach, and a market-based approach, evaluated in a distributed sensing scenario. Simulation results compare the three approaches in scalability and heterogenity, and show that all three approaces perform well with heterogeneous teams, and the market method performs best overall in scalability. Rabideau et al. [19] also conduct a similar comparative study between a centralized planner that does not guarantee optimality, a distributed planner, and a single-task auction approach. They conclude that the auction approach performs best but takes up the most CPU cycles. Although market-based approaches have performed well in comparative studies, these studies are fairly limited and broader studies are in high demand.

A final challenge is to demonstrate long-term, reliable, and robust operation of larger robot teams. One requisite of this goal is the effective use of learning techniques. The application of learning techniques in market-based coordination is currently at a very early stage. One big debate is whether learning should be applied at the team level, at the individual level, or some combination of the two. Oliveira et al. [51] present a detailed discussion of the issues relevant to the application of learning in dynamic markets. The role that learning can play in market-based multirobot coordination is also discussed briefly by Stentz and Dias [52]. The authors are unaware of any learning techniques implemented on a team of physical robots coordinated using a market-based approach. However, publications are starting to emerge in the application of learning techniques for market-based coordination of simulated robot teams. Notably, learning techniques are applied to learn bidding strategies in dynamic markets [51], opportunity costs in a simulated distributed sensing task [23], and role assignment [13] and bidding strategies [53] in simulated robot soccer.

Despite the many challenges ahead, market-based techniques are proving to be versatile and powerful coordination schemes for groups of robots executing complex tasks as part of a team. Different application requirements 
and tradeoffs in implementation make it difficult to construct a single market-based approach that can be successful in all domains. In fact, the authors are aware of only two market-based approaches, MURDOCH [7] and TraderBots [17], [20], [31], that have been demonstrated in more than one application domain. Nevertheless, a welldesigned market-based approach with sufficient plug-andplay options for manually or automatically altering different tradeoffs can be successful in a wide range of applications. And, with further research, market-based approaches promise to significantly further our vision of robots playing an integral role in human life.

\section{Acknowledgment}

The views and conclusions contained in this document are those of the authors and should not be interpreted as representing the official policies, either expressed or implied, of the Boeing Company, the Army Research Laboratory, the U.S. Government, or the Qatar Foundation.

\section{REFERENCES}

[1] D. J. Farber and K. C. Larson, "The structure of a distributed computing systemSoftware," in Proc. Symp. ComputerCommunications Networks and Teletraffic, 1972, pp. 101-108.

[2] R. G. Smith, "The contract net protocol: High-level communication and control in a distributed problem solver," IEEE Trans. Comput., vol. C-29, no. 12, pp. 1104-1113, Dec. 1980.

[3] A. Pongpunwattana, R. Rysdyk, J. Vagners, and D. Rathbun, "Market-based co-evolution planning for multiple autonomous vehicles," in Proc. 2nd AIAA Unmanned Unlimited Systems, Technologies and Operations Conf., 2003.

[4] E. Wolfstetter, "Auctions: An introduction," J. Economic Surveys, vol. 10, no. 4, pp. 367-420, 1996.

[5] T. Sandholm, "Algorithm for optimal winner determination in combinatorial auctions," Artif. Intell., vol. 135, no. 1, pp. 1-54, 2002.

[6] B. P. Gerkey and M. J. Mataric, "A formal analysis and taxonomy of task allocation in multi-robot systems," Int. J. Robot. Res. vol. 23, no. 9, pp. 939-954, 2004.

[7] B. P. Gerkey and M. J. Matarić, "Sold!: Auction methods for multi-robot control," IEEE Trans. Robot. Autom. (Special Issue on Multi-Robot Systems), vol. 18, no. 5, pp. 758-768, Oct. 2002.

[8] M. B. Dias, M. Zinck, R. Zlot, and A. Stentz, "Robust multirobot coordination in dynamic environments," in Proc. IEEE Int. Conf. Robotics and Automation (ICRA), 2004, pp. 3435-3442.

[9] M. Lagoudakis, E. Markakis, D. Kempe, P. Keskinocak, A. Kleywegt, S. Koenig, C. Tovey, A. Meyerson, and S. Jain, "Auction-based multi-robot routing," Robot. Sci. Syst., 2005.

[10] M. B. Dias, R. Zlot, M. Zinck, J. P. Gonzalez, and A. Stentz, "A versatile implementation of the TraderBots approach to multirobot coordination," in Proc. Int. Conf. Intelligent Autonomous Systems (IAS), 2004, pp. 325-334.

[11] N. Kalra, D. Ferguson, and A. Stentz, "Hoplites: a market-based framework for complex tight coordination in multi-robot teams," in Proc. IEEE Int. Conf. Robotics and Automation (ICRA), 2005, pp. 1170-1177.

[12] M. Andersson and T. Sandholm, "Contract type sequencing for reallocative negotiation," in Proc. Int. Conf. Distributed Computing Systems, 2000, pp. 154-160.

[13] H. Köse, U. Tatlidede, Ç. Meriçli, K. Kaplan, and H. L. Akin, "Q-learning based market-driven multi-agent collaboration in robot soccer," in Proc. Turkish Symp. Artificial Intelligence and Neural Networks, 2004, pp. 219-228.
[14] R. Simmons, D. Apfelbaum, W. Burgard, D. Fox, M. Moors, S. Thrun, and H. Younes, "Coordination for multi-robot exploration and mapping," in Proc. National Conf. Artificial Intelligence (AAAI), 2000, pp. 851-858.

[15] D. Vail and M. Veloso, "Multi-robot dynamic role assignment and coordination through shared potential fields," in Multi-Robot Systems: From Swarms to Intelligent Automata: Proc. 2003 Int. Workshop Multi-Robot Systems, vol. 2, A. Schultz, L. Parker, and F. Schneider, Eds., 2003, pp. 87-98.

[16] M. Berhault, H. Huang, P. Keskinocak, S. Koenig, W. Elmaghraby, P. Griffin, and A. Kleywegt, "Robot exploration with combinatorial auctions," in Proc. IEEE/RS Int. Conf. Intelligent Robots and Systems (IROS), 2003, pp. 1957-1962.

[17] M. B. Dias, “Traderbots: A new paradigm for robust and efficient multirobot coordination in dynamic environments," Ph.D. dissertation, Robotics Inst., Carnegie Mellon Univ., Pittsburgh, PA, Jan. 2004.

[18] M. Golfarelli, D. Maio, and S. Rizzi, "A Task-Swap negotiation protocol based on the contract net paradigm," Research Center for Informatics and Telecommunication Systems (CSITE), Univ. Bologna, Tech. Rep. 005-97, 1997.

[19] G. Rabideau, T. Estlin, S. Chien, and A. Barrett, "A comparison of coordinated planning methods for cooperating rovers," in Proc. AIAA 1999 Space Technology Conf., 1999.

[20] R. Zlot, A. Stentz, M. B. Dias, and S. Thayer, "Multi-robot exploration controlled by a market economy," in Proc. IEEE Int. Conf. Robotics and Automation (ICRA), 2002, pp. 3016-3023.

[21] D. Goldberg, V. Cicirello, M. B. Dias, R. Simmons, S. Smith, and A. Stentz, "Market-based multi-robot planning in a distributed layered architecture," in Multi-Robot Systems: From Swarms to Intelligent Automata: Proc. 2003 Int. Workshop Multi-Robot Systems, vol. 2, A. Schultz, L. Parker, and F. Schneider, Eds., 2003, pp. 27-38.

[22] T. Lemaire, R. Alami, and S. Lacroix, "A distributed tasks allocation scheme in multi-UAV context," in Proc. IEEE Int. Conf. Robotics and Automation (ICRA), 2004, pp. 3622-3627.

[23] J. Schneider, D. Apfelbaum, D. Bagnell, and R. Simmons, "Learning opportunity costs in multi-robot market based planners," in Proc. IEEE Int. Conf. Robotics and Automation (ICRA), 2005, pp. 1151-1156.

[24] J. Guerrero and G. Oliver, "Multi-robot task allocation strategies using auction-like mechanisms," in Proc. 6th Congr. Catalan Association for Artificial Intelligence (CCIA), 2003, pp. 111-122.
[25] L. Lin and Z. Zheng, "Combinatorial bids based multi-robot task allocation method," in Proc. IEEE Int. Conf. Robotics and Automation (ICRA), 2005, pp. 1145-1150.

[26] E. G. Jones, B. Browning, M. B. Dias, B. Argall, M. Veloso, and A. Stentz, "Dynamically formed heterogeneous robot teams performing tightly-coupled tasks," in Proc. IEEE Int. Conf. Robotics and Automation (ICRA), 2006, pp. 570-575.

[27] S. S. C. Botelho and R. Alami, "M+: A scheme for multi-robot cooperation through negotiated task allocation and achievement," in Proc. IEEE Int. Conf. Robotics and Automation (ICRA), 1999, pp. 1234-1239.

[28] D. C. MacKenzie, "Collaborative tasking of tightly constrained multi-robot missions," in Multi-Robot Systems: From Swarms to Intelligent Automata: Proc. 2003 Int. Workshop Multi-Robot Systems, vol. 2, pp. 39-50.

[29] M. B. Dias, B. Browning, M. M. Veloso, and A. Stentz, "Dynamic heterogenous robot teams engaged in adversarial tasks," Robotics Inst., Carnegie Mellon Univ., Pittsburgh, PA, Tech. Rep. CMU-RI-TR-05-14, 2005.

[30] P. Caloud, W. Choi, J. C. Latombe, C. L. Pape, and M. Yim, "Indoor automation with many mobile robots," in Proc. IEEE/RSJ Int. Conf. Intelligent Robots and Systems (IROS), 1990, pp. 67-72.

[31] R. Zlot and A. Stentz, "Market-based multirobot coordination for complex tasks," in Int. J. Robot. Res. (Special Issue on 5th Int. Conf. Field and Service Robotics), vol. 25, no. 1, pp. 73-101, Jan. 2006.

[32] K. Azarm and G. Schmidt, "A decentralized approach for the conflict free motion of multiple mobile robots," in Proc. IEEE/RSJ Int. Conf. Intelligent Robots and Systems (IROS), 1996, pp. 1667-1674.

[33] R. Simmons, S. Singh, D. Hershberger, J. Ramos, and T. Smith, "First results in the coordination of heterogeneous robots for large-scale assembly," in Proc. Int. Symp. Experimental Robotics (ISER), 2000, pp. 323-332.

[34] R. Nair, T. Ito, M. Tambe, and S. Marsella, "Task allocation in the rescue simulation domain: A short note," in Proc. RoboCup-2001: Fifth Robot World Cup Games and Conf., 2002, pp. 751-754.

[35] T. Sandholm, "An implementation of the contract net protocol based on marginal cost calculations," in Proc. 12th Int. Workshop Distributed Artificial Intelligence, 1993, pp. 295-308.

[36] C. Tovey, M. G. Lagoudakis, S. Jain, and S. Koenig, "The generation of bidding rules for auction-based robot coordination," in Proc. 3rd Int. Multi-Robot Systems Workshop, 2005, pp. 3-14. 
[37] M. B. Dias, B. Ghanem, and A. Stentz, "Improving cost estimation in market-based coordination of a distributed sensing task," in Proc. IEEE/RSJ Int. Conf. Intelligent Robots and Systems (IROS), 2005, pp. 3972-3977.

[38] P. Chandler and M. Pachter, "Hierarchical control for autonomous teams," in Proc. AIAA Guidance, Navigation, and Control Conf., 2001, pp. 632-642.

[39] B. Kalyanasundaram and K. Pruhs, "Online weighted matching," J. Algorithms, vol. 14, no. 3, pp. 478-488, 1993.

[40] L. Hunsberger and B. J. Grosz, "A combinatorial auction for collaborative planning," in Proc. 4th Int. Conf. Multi-Agent Systems (ICMAS), 2000, pp. 151-158.

[41] M. B. Dias and A. Stentz, "Opportunistic optimization for market-based multirobot control," in Proc. IEEE/RSJ Int. Conf. Intelligent Robots and Systems (IROS), 2002, pp. 2714-2720.

[42] T. Sandholm, "Contract types for satisficing task allocation: I theoretical results," in Proc. AAAI Spring Symp.: Satisficing Models, 1998, pp. 68-75.
[43] J. M. Vidal, "The effects of cooperation on multiagent search in task-oriented domains," in Proc. Autonomous Agents and Multi-Agent Systems Conf., 2002, pp. 453-454.

[44] M. B. Dias, D. Goldberg, and A. Stentz, "Market-based multirobot coordination for complex space applications," in 7th Int. Symp. Artificial Intelligence, Robotics and Automation in Space (i-SAIRAS), 2003.

[45] J. O. Cerdeira, "The multi-depot traveling salesman problem," Investigação Operacional, vol. 12, no. 2, pp. 159-167, 1992.

[46] B. L. Golden and A. A. Assad, Eds. Vehicle Routing: Methods and Studies (Studies in Management Science and Systems). Amsterdam: Elsevier, 1988, vol. 16.

[47] G. Laporte, Y. Nobert, and H. Mercure, "The multi-depot travelling salesman problem," Methods Operations Res., vol. 40, pp. 367-376, 1981.

[48] C. Bererton, G. Gordon, S. Thrun, and P. Khosla, "Auction mechanism design for multi-robot coordination," in Proc. Advances in Neural Information Processing Systems Conf., 2003, pp. 879-886.
[49] V. Frias-Martinez, E. Sklar, and S. Parsons, "Exploring auction mechanisms for role assignment in teams of autonomous robots,' in Proc. RoboCup Symp., 2004, pp. 532-539.

[50] N. Kalra, R. Zlot, M. B. Dias, and A. Stentz, "Market-based multirobot coordination: A comprehensive survey and analysis," Robotics Inst., Carnegie Mellon Univ. Pittsburgh, PA, Tech. Rep. CMU-RITR-05-16, 2005

[51] E. Oliveira, J. M. Fonseca, and N. R. Jennings, "Learning to be competitive in the market," in Proc. AAAI Workshop Negotiation: Settling Conflicts and Identifying Opportunities, 1999, pp. 30-37.

[52] A. Stentz and M. B. Dias, A Free Market Architecture for Coordinating Multiple Robots, Robotics Inst., Carnegie Mellon Univ., Pittsburgh, PA, Tech. Rep. CMU-RI-TR99-42, Dec. 1999.

[53] V. Frias-Martinez and E. Sklar, "A team-based co-evolutionary approach to multi agent learning," in Proc. 2004 AAMAS Workshop Learning and Evolution in Agent Based Systems, 2004.

\section{ABOUT THE AUTHORS}

M. Bernardine Dias received the Ph.D. degree in robotics from Carnegie Mellon University, Pittsburgh, PA, in 2004.

She is a Research Scientist with the Robotics Institute at Carnegie Mellon University. Her thesis work developed the TraderBots market-based framework for efficient multirobot coordination in dynamic environments. Her research interests include team coordination in unstructured environments and technology relevant to developing communities.

Robert Zlot received the B.S. degree in space and communication science from York University, Toronto, ON, Canada, in 2000 and the M.S. degree in robotics from Carnegie Mellon University, Pittsburgh, PA, in 2002. He is currently working toward the Ph.D. degree at the Robotics Institute at Carnegie Mellon University.

He has been working with market-based multirobot systems since 2000. His current research focus is on multirobot coordination for complex, structured tasks.

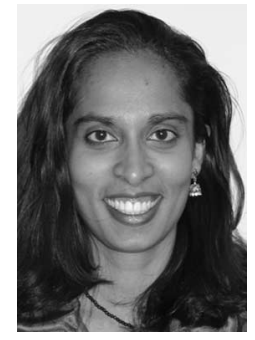

Nidhi Kalra received the B.S. degree in computer science with a concentration in electrical engineering from the College of Engineering, Cornell University, Ithaca, NY, in 2002 and the M.S. degree in robotics from Carnegie Mellon University, Pittsburgh, PA, in 2004. She is currently working toward the Ph.D. degree at the Robotics Institute at Carnegie Mellon University.

Her current research focuses on planned tight coordination of multirobot teams.

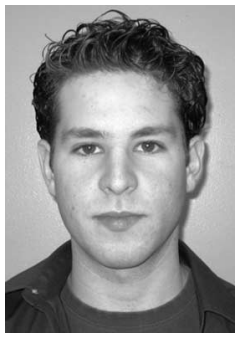

Anthony Stentz received the Ph.D. degree in computer science from Carnegie Mellon University, Pittsburgh, PA, in 1989.

$\mathrm{He}$ is a Research Professor and Associate Director of the National Robotics Engineering Consortium at the Robotics Institute, Carnegie Mellon University. His research expertise includes multivehicle planning and coordination (for which he has executed programs for NASA, DARPA, and the Army with ground and air vehicles), dynamic path planning, and autonomous vehicle navigation.
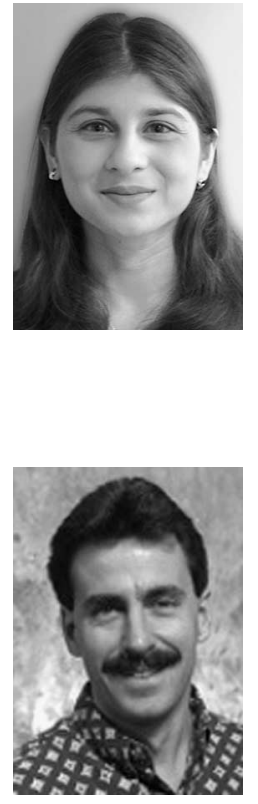